\title{
Short cracks observations on surfaces of specimens made of three materials, subjected to synchronous and asynchronous multiaxial loadings
}

\author{
Eukasz Pejkowski ${ }^{1}$, Jan Seyda $^{1}$, and Dariusz Skibicki ${ }^{1,1}$ \\ ${ }^{1}$ UTP University of Science and Technology in Bydgoszcz, Kaliskiego 7, 85-796 Bydgoszcz, Poland
}

\begin{abstract}
In this paper analysis of short cracks observed on surfaces of specimens subjected to axial, torsional, synchronous in-phase and out-ofphase as well as a number of asynchronous loadings was performed. The tests were conducted on PA38-T6 aluminum alloy, E235 non-alloy steel and 1.4301 austenitic stainless steel thin-walled specimens, in strain-control mode. It was concluded that for PA38-T6 aluminum alloy and E235 steel, fatigue cracks initiated and propagated on the plane of maximum shear strain, regardless of the loading case. Fatigue cracks observed on the surface of 1.4301 steel specimens developed predominantly on the levels of maximum normal strain. For this material, the surface condition resulting from the machining had a significant impact on the cracks growth. In the case of non-proportional loadings, at high levels of strain, cracks often propagated along the machining marks.
\end{abstract}

\section{Introduction}

Short cracks are defined as measuring less than $1 \mathrm{~mm}$ in length $[1,2]$. Their analysis is important because the process of initiation and growth of short cracks constitutes the majority of fatigue life of the specimens. The observations made during the research show that the occurrence of macro-cracks takes place in just the last few loading cycles. Moreover, Socie stated that based on the observation of the damage mechanism of a given material, the choice of the fatigue model should be made [3].

In this paper an analysis of short cracks observed on the surface of specimens made of PA38 aluminum alloy, E235 steel and 1.4301 steel has been performed. The subjects of the analysis were: a) determination of damage mechanism based on crack direction observation, and b) correlation of damage mechanism with multiaxial fatigue models, especially for the asynchronous loadings.

\section{Materials and methods}


Materials and experimental procedure were already described in detail in [4, 5]. Fatigue tests were performed on thin-walled cylindrical specimens in strain-controlled mode on an Instron 8874 axial-torsional closed-loop servo-hydraulic testing system. Specimens were made of PA38-T6 (AW 6060-T6) aluminum alloy, solution heat-treated and artificially aged, E235 (1.0308) non-alloy quality steel, normalized, and 1.4301 (X5CrNi18-10, 304) austenitic stainless steel, soft annealed.

In case of fully reversed sine-shaped loadings, which were applied in this study, the waveforms of controlled normal $\varepsilon$ and shear $\gamma$ strains can be written as:

$$
\begin{gathered}
\varepsilon(t)=\varepsilon_{a} \sin \left(2 \pi f_{\varepsilon} t\right) \\
\gamma(t)=\gamma_{a} \sin \left(2 \pi f_{\gamma} t+\delta\right)
\end{gathered}
$$

where $\varepsilon_{a}$ and $\gamma_{a}$ are amplitudes, $f_{\varepsilon}$ and $f_{\gamma}$ are frequencies of normal and shear strain, respectively, and $\delta$ is a phase shift angle. The parameters of loadings applied in case of individual materials are listed in Table 1. First, four basic synchronous loadings were utilized: tension-compression (TC), torsion (TOR), in-phase loading (IP) and $90^{\circ}$ out-of-phase loading. Then, a number of asynchronous loadings (ASN) with different parameters were applied.

Table 1. Parameters of loadings applied with regard to individual materials.

\begin{tabular}{|c|c|c|c|c|}
\hline Loading case & $\boldsymbol{\lambda}$ & $\boldsymbol{\delta}$, degress & $\boldsymbol{f}_{\boldsymbol{\gamma}} / \boldsymbol{f}_{\boldsymbol{\varepsilon}}$ & Material \\
\hline TC & 0 & 0 & 1 & PA38-T6, E235, 1.4301 \\
\hline TOR & $\infty$ & 0 & 1 & PA38-T6, E235, 1.4301 \\
\hline IP & $\sqrt{3}$ & 0 & 1 & PA38-T6, E235, 1.4301 \\
\hline OP & $\sqrt{3}$ & 90 & 1 & PA38-T6, E235, 1.4301 \\
\hline ASN1 & $\sqrt{3}$ & 0 & 0.5 & PA38-T6, E235, 1.4301 \\
\hline ASN2a & $\sqrt{3} / 2$ & 0 & 4 & PA38-T6, E235, 1.4301 \\
\hline ASN3a & $2 \sqrt{3}$ & 0 & 0.2 & PA38-T6, 1.4301 \\
\hline ASN3b & $2 \sqrt{3}$ & 0 & 0.25 & E235 \\
\hline ASN4a & $\sqrt{3} / 2$ & 0 & $2 / 3$ & E235 \\
\hline ASN4b & $3 \sqrt{3} / 5$ & 0 & 6 & 1.4301 \\
\hline ASN4c & $\sqrt{3}$ & 0 & 8 & PA38-T6 \\
\hline ASN5 & $\sqrt{3}$ & 0 & 0.7 & PA38-T6, E235, 1.4301 \\
\hline
\end{tabular}

In the present study short cracks observed on the surfaces of the specimens were analyzed. Observations were performed on failed specimens. Crack photos were taken on a metallographic optical microscope equipped with a digital camera. A scale and graphs showing the distribution of the maximum normal $\varepsilon_{\max }(\varphi)$ and shear $\gamma_{\max }(\varphi)$ strain on the plane, whose position in the polar coordinate system is given by the angle $\varphi$, were plotted on the photos [6]. The angle $\varphi=0^{\circ}$ corresponds to the position of the specimen axis. The images of fatigue cracks representative for a given type of load were presented.

\section{Cracks observations}

\subsection{PA38-T6 aluminum alloy}

Typical short cracks observed on the surfaces of specimens made of PA38-T6 aluminum alloy were presented in Fig. 1 for all the applied loading cases. 
For TC loading it can be observed that macrocrack occurs due to the coalescence of short cracks, oriented at approximately $45^{\circ}$ and $135^{\circ}$ angles. These directions coincide with the directions of maximum shear strain planes.

In the case of TOR loading, the observed cracks are located at an angle of $0^{\circ}$ and $90^{\circ}$, which in this case corresponds to the location of maximum shear strain planes. Macrocracks were oriented along the specimen's axis, which is characteristic for torsional loading with a high level of strain, in the case of materials exhibiting a shear damage mechanism $[1,7]$.

In the case of IP loading, short fatigue cracks can be observed located at an angle of approximately $70^{\circ}$ and $160^{\circ}$. These directions are consistent with the location of the maximum shear strain planes too.

Regarding the OP loading, short cracks occur in many directions, because high-value shear strains occur on all planes. However, cracks located at a $90^{\circ}$ angle are much more developed. This plane is also subjected to the maximum normal strain causing the crack opening, which in turn results in a reduction of friction between the fracture surfaces. This phenomenon accelerates the development of fatigue cracks. Macrocracks are also formed from these cracks.

For asynchronous loadings only selected pictures of crack were presented in this study. In case of all ASN loading cases extreme values of shear strain occurs on four directions, located at $22^{\circ}, 68^{\circ}, 112^{\circ}$ and $158^{\circ}$ for ASN1, $30^{\circ}, 60^{\circ}, 120^{\circ}$ and $150^{\circ}$ for ASN2a, $78^{\circ}, 168^{\circ}$, $11^{\circ}$ and $101^{\circ}$ for ASN3a, $20^{\circ}, 70^{\circ}, 110^{\circ}$ and $160^{\circ}$ for ASN4c and $20^{\circ}, 70^{\circ}, 110^{\circ}$ and $160^{\circ}$ for ASN5 loading. Short cracks usually occur in all of these directions, but in general, cracks are most developed at planes on which normal strains with a higher value also act.

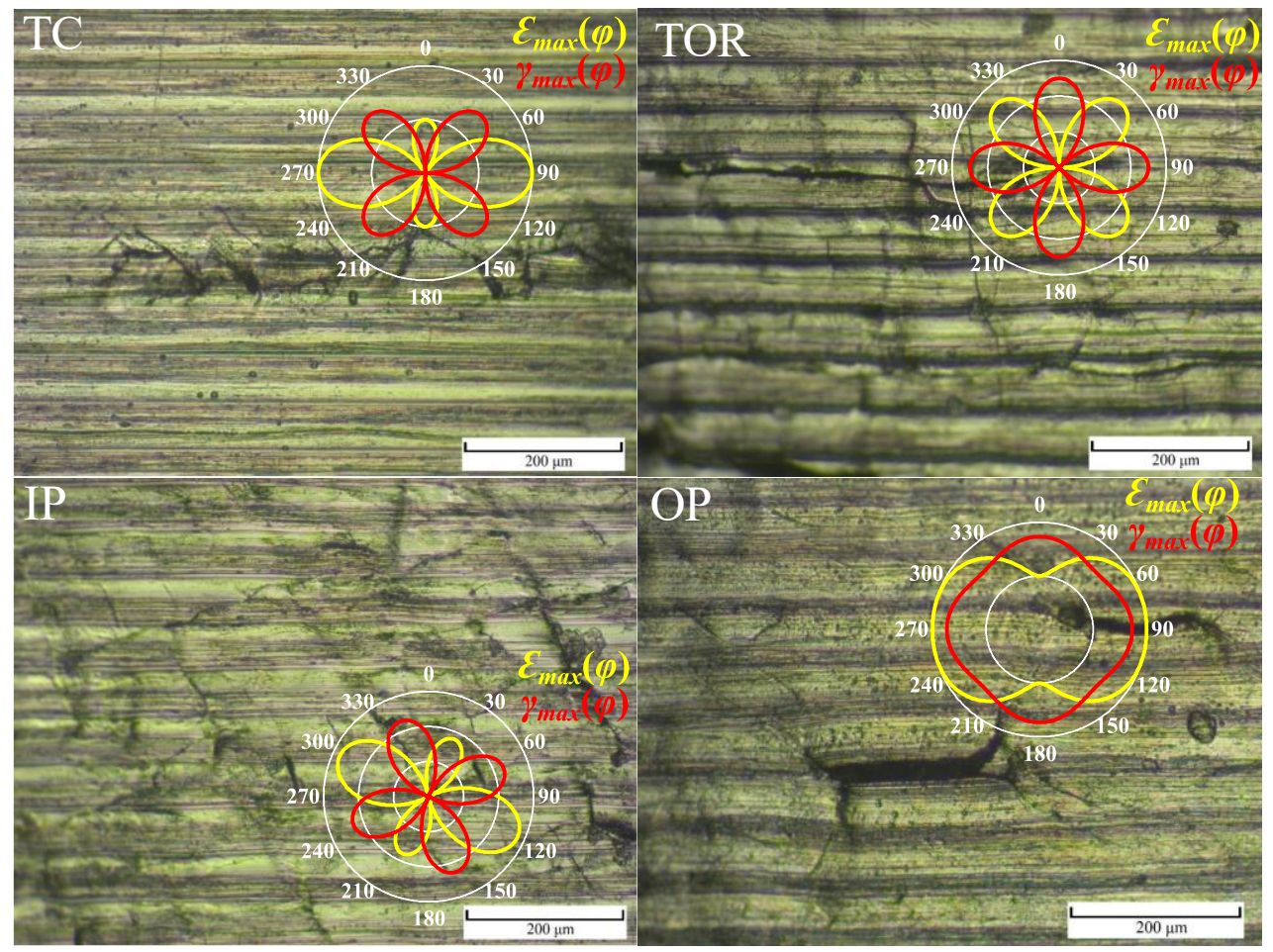




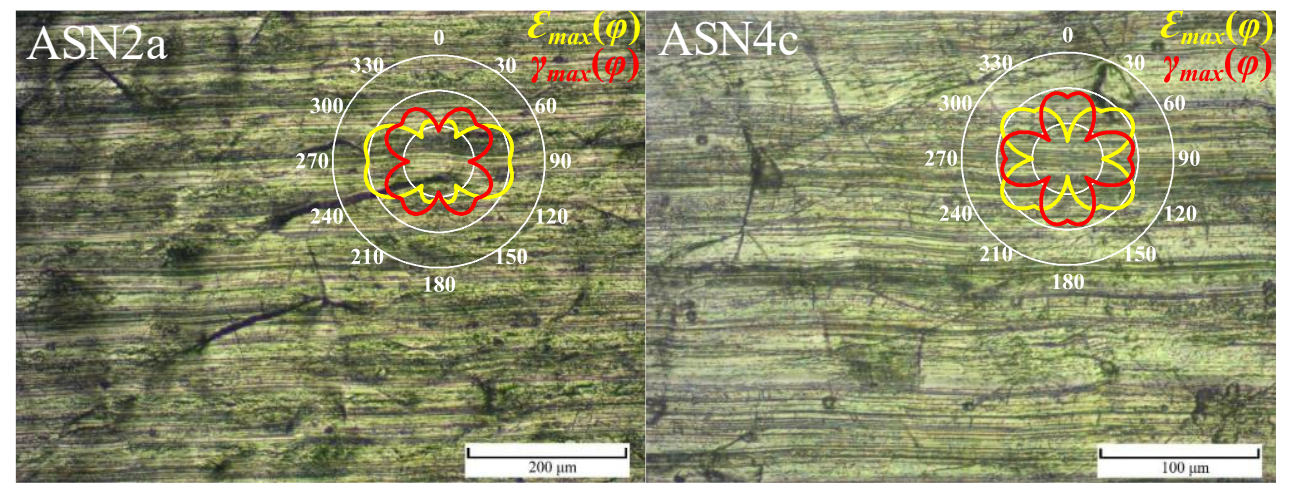

Fig. 1. Typical short cracks observed on the surfaces of PA38-T6 aluminum alloy specimens.

\subsection{E235 steel}

Pictures of fatigue cracks present on the surfaces taken in case of E235 steel specimens are presented in Fig. 2.

In the case of TC loading on the surfaces of the specimens, numerous fatigue cracks were observed at an angle of $45^{\circ}$ and $135^{\circ}$. These are the directions of maximum shear strains.

Specimens subjected to TOR loading were characterized by cracks in the direction of $0^{\circ}$ and $90^{\circ}$. For this loading case, these are directions of maximum shear strains.

In the case of IP loading, most cracks are located at an angle of $70^{\circ}$. In this direction, cracks of greater length also occur. In the direction of $160^{\circ}$, cracks can also be observed, but they are much shorter. Both of these directions coincide with the directions on which the maximum shear strains acts.

In the case of OP loading, fatigue cracks were oriented in the $90^{\circ}$ direction. This is the plane on which both the maximum shear and maximum normal strains occur.

For asynchronous loadings, pictures for selected loading cases are presented. Maximum shear strain directions are: $22^{\circ}, 68^{\circ}, 112^{\circ}$ and $158^{\circ}$ for ASN1, $30^{\circ}, 60^{\circ}, 120^{\circ}$ and $150^{\circ}$ for ASN2a, $11^{\circ}, 79^{\circ}, 110^{\circ}$ and $168^{\circ}$ for ASN3b and $30^{\circ}, 60^{\circ}, 120^{\circ}$ and $150^{\circ}$ for ASN4a loading. Just like in the case of PA38-T6 aluminium alloy, cracks developed in directions of maximum shear strains. Those of them, for which normal strain was also high are more developed. It was noticed that depending on the location on the surface of the specimen, cracks in one direction were more developed than in others, but the distribution was random.

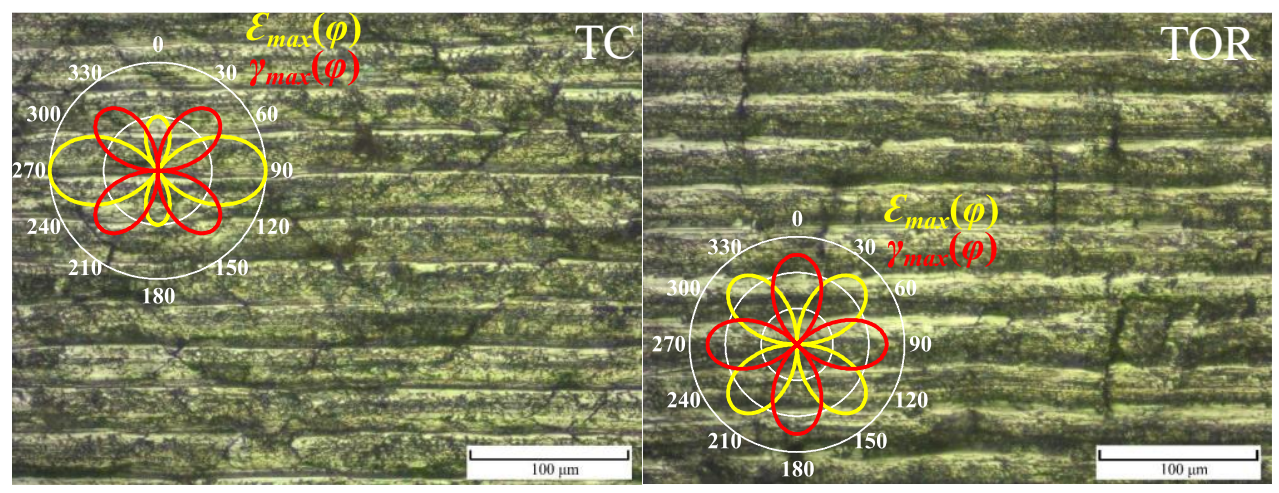




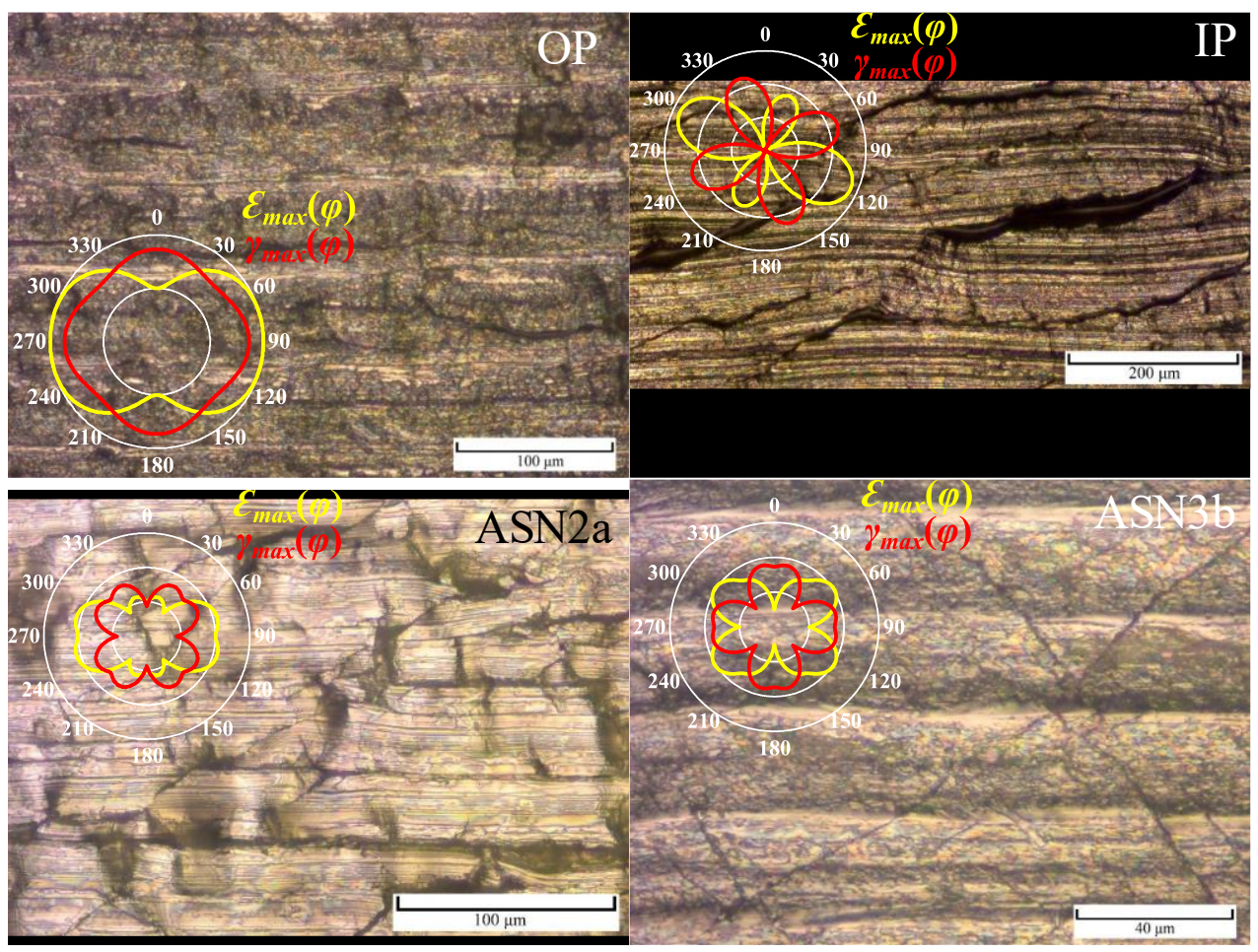

Fig. 2. Typical short cracks observed on the surfaces of E235 steel specimens.

\subsection{1 .4301 steel}

Under TC loading two stages of cracks propagation can be noticed (Fig. 3). In the first stage, the crack developed at an angle of $45^{\circ}$, i.e. towards the maximum shear strain. Further development occurred towards the maximum normal strain. The fragment of the crack developed in this direction is longer.

In the case of loading the TOR, the crack also initiated and initially propagated in the direction of the maximum shear strain, followed by its branching and further propagation towards the maximum normal strain. Many cracks of this type have been observed on the entire surface. There are many such cracks at a low level of load, while at a higher load level, fewer cracks have been observed that have developed to larger sizes.

In the case of IP loading, fatigue cracks located at an angle of $70^{\circ}$ and $160^{\circ}$, as well as $115^{\circ}$, can be seen. The first two angles correspond to the position of the plane of maximum shear strains, while the angle of $115^{\circ}$ corresponds to the plane of the maximum normal strain.

Under the OP loading, the positions of the plane of the maximum shear and normal strain are the same. This is a plane at $90^{\circ}$. Fatigue cracks observed for this loading case always developed at the bottom of the machining marks, which is also located at an angle of $90^{\circ}$. Macro crack occurred in this direction due to the merging of short cracks formed in the bottom of the same machining mark.

For ASN1 loading, the longest fragments of fatigue cracks are located in the bottom of the machining marks, as in the case of OP loading, although there are no strain extremes in this direction. In some cases, under a low loading level, very short fragments of cracks were also observed, the direction of which coincides with the direction of maximum shear strains. Presumably, the fatigue crack was initiated and initially propagated towards the maximum 
shear strain direction, similar to the previously discussed loadings, after which further propagation took place in the bottom of the trace machining marks.

Very similar behaviour of fatigue cracks was noted for all other cases of asynchronous loadings. For ASN2a loading cracks also propagated mainly along the machining marks, although at a low level of strain, fragments were also observed whose direction is consistent with the location of the maximum shear strain planes. Very short cracks can also be found on the surface of the samples, the direction of which is consistent with the direction of the maximum shear strain.

In the case of ASN3a and ASN4b loadings, for a high loading level, cracks were observed, which propagated in the direction perpendicular to the axis of the specimen, along with the machining marks. These cracks then merged to form macrocracks. For low loading levels, very short cracks can be noticed, the direction of which is generally consistent with the location of the maximum normal strain planes.

Similarly, on the surface of specimens subjected to ASN5 loading, cracks propagated along the machining marks for high loading levels. In the case of lower levels of loading, short cracks developed towards the extremes of normal and shear strains are noticeable, which in this case are the same. Most often, slightly more developed cracks were observed for the direction on which normal strains a higher value.

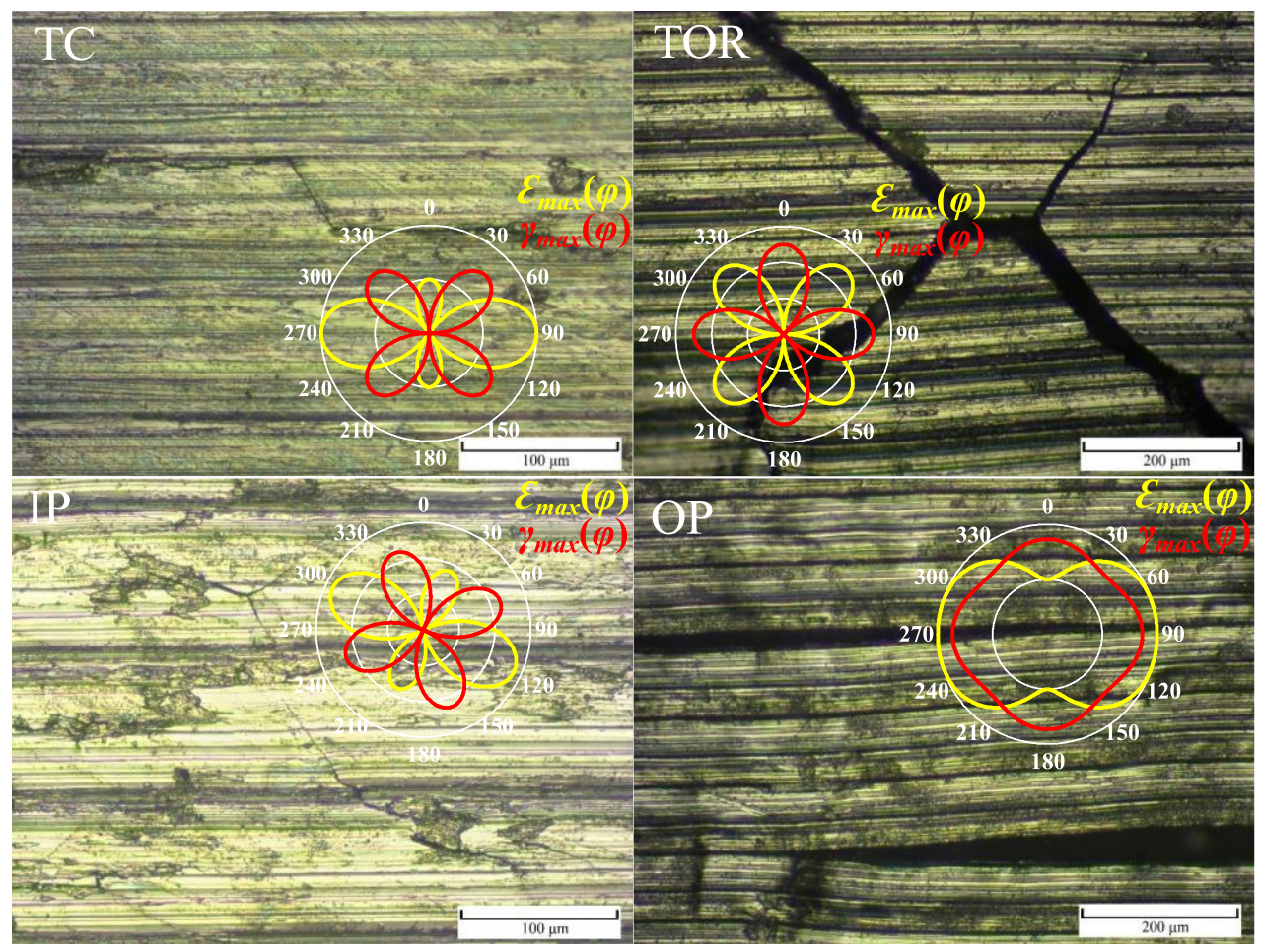




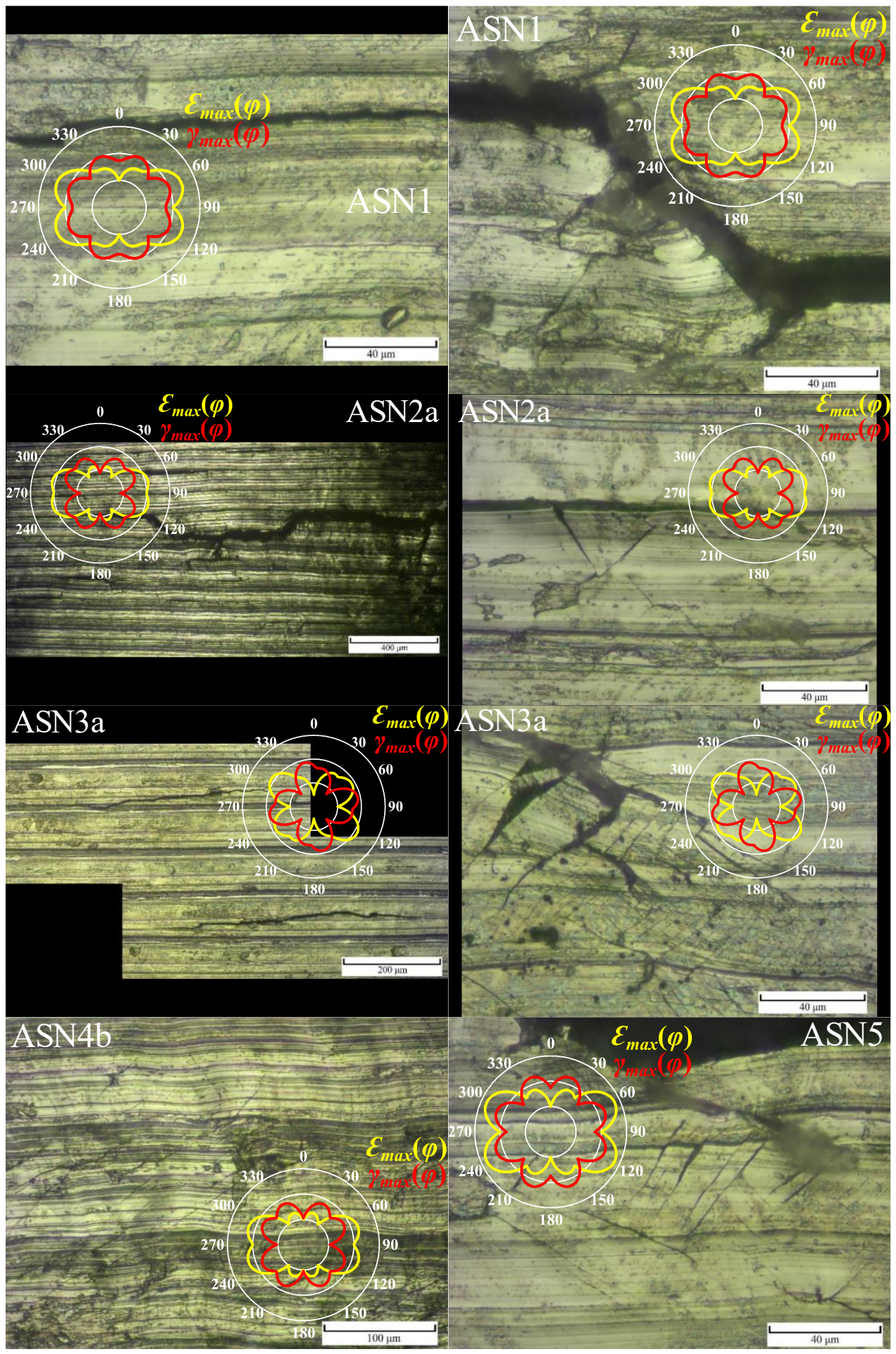

Fig. 3. Typical short cracks observed on the surfaces of 1.4301 steel specimens. 


\section{Discussion and conclusions}

In the case of PA38-T6 aluminum alloy and E235 steel, fatigue cracks initiated and propagated on the plane of maximum shear strain, regardless of the loading case. If the maximum or near-maximum values of the shear occurred on many planes, short cracks can be observed in all of these directions. However, cracks are more developed on planes for which also large positive values of normal strain occurred, causing crack opening.

Fatigue cracks observed on the surface of 1.4301 steel specimens developed predominantly on the levels of maximum normal strain (planes of principal strains). For some cases and loading levels, very short parts of cracks can be observed, the direction of which is consistent with the direction of the plane of maximum shear strain. However, these cracks branched towards the maximum normal strain after reaching a certain length. For this material, the surface condition resulting from the machining had a significant impact on the cracks growth. In the case of non-proportional loadings, at high levels of strain, cracks often propagated along the machining marks. Potential reasons for this behavior include the stresses concentration in the machining marks roots and the martensitic transformation taking place at these points. It was also observed that in case of asynchronous loadings, fatigue cracks were often initiated in the vicinity of inclusions visible on the surface of the material. These inclusions probably occurred due to the formation of a built-up edge on the cutting tool.

Taking into account the above observations, it could be assumed that for the first two materials the more accurate should be the models based on the maximum shear strain plane, like Brown-Miller, Fatemi-Socie or Glinka-Wang-Plumtree, whereas in case of 1.4301 steel, the maximum normal strain-based models, e.g. SWT, is expected to give better results.

\section{References}

1. Socie, D., Marquis, G.: Multiaxial Fatigue. SAE International, United States (2000).

2. Shamsaei, N., Fatemi, A.: Small fatigue crack growth under multiaxial stresses. Int. J. Fatigue. 58, 126-135 (2014).

3. Socie, D.: Critical plane approaches for multiaxial fatigue damage assessment. ASTM Spec. Tech. Publ. 1191, 7-36 (1993).

4. Skibicki, D., Pejkowski, Ł.: The relationship between additional non-proportional hardening coefficient and fatigue life. Int. J. Fatigue. 123, 66-78 (2019).

5. Pejkowski, Ł., Skibicki, D.: Fatigue behavior of selected materials under multiaxial asynchronous loadings. In: The 12th International Conference on Multiaxial Fatigue and Fracture., Bordeaux (2019).

6. Albinmousa, J.: Investigation on multiaxial fatigue crack path using polar stressstrain representation. Int. J. Fatigue. 92, 406-414 (2016).

7. Kurath, P., Socie, D.F.: The Relationship Between Observed Fatigue Damage and Life Estimation Models. , Urbana (1988). 